\title{
A Validation of the Viscous Dissipation Model for Fast Vortex Methods in Simulations of Decaying Turbulence*
}

\author{
Yoshitaka TOTSUKA** and Shinnosuke OBI** \\ ** Department of Mechanical Engineering, Keio University, \\ 3-14-1 Hiyoshi, Kouhoku-ku, Yokohama 223-8522, Japan \\ E-mail: obsn@mech.keio.ac.jp
}

\begin{abstract}
For realistic turbulent flow simulations, quantitative representation of turbulent flow dynamics is desired. In the present study, two-dimensional homogeneous isotropic turbulence is simulated by using a grid-free vortex method to focus on the viscous dissipation process. The results are compared with those of spectral DNS. Two viscous diffusion models, i.e., a core spreading model and Moving Particle Semi-implicit(MPS) Laplacian model, are compared. For the former model, merging and insertion of particles are incorporated to ensure uniform distribution of vortex elements. It is shown that the MPS Laplacian model is superior to the conventional core spreading model in terms of the decay rate of enstrophy and energy spectra. Furthermore, the computational time is remarkably reduced by using a Fast Multipole Method(FMM), while retaining accuracy.
\end{abstract}

Key words : Turbulent Flow, Computational Fluid Dynamics, Vortex, Grid-Free Vortex Method, Viscous Dissipation Process, MPS, FMM

\section{Introduction}

Grid-free Vortex Methods have many advantages in calculating turbulent flows of engineering interest. For example, it can easily represent complex geometries; thus, it is suitable for moving boundary problems and fluid structure interaction problems. Furthermore, it may have an advantage in solving exterior flows, where the vorticity is confined to the surface of the body, since it is only necessary to solve where the vorticity exists. Recently, studies of an unsteady flow simulation around a wind turbine blade ${ }^{(1)}$ have been reported.

In engineering applications it is crucial that the conservation laws are satisfied. However, there have been few attempts to scrutinize the energy dissipation process of vortex methods for turbulent flows. Vortex methods for turbulent flows have mainly focused on the interaction of vortex rings ${ }^{(2)}$, where the energy spectrum of the inertial subrange was successfully calculated. For viscous diffusion there have been reports about calculations of the homogeneous isotropic turbulence by the vortex-in-cell method ${ }^{(3)}$. However, there have been no reports about a quantitative analysis of the energy dissipation in the context of grid-free vortex methods. Consequently, it is necessary to individually validate the energy cascade, viscous diffusion, and numerical diffusion systematically.

As a first step to various issues, that mast be solved before the vortex method is applied to various turbulent flows, the present study focuses on the diffusion models which are commonly used in the current framework. In particular, the representation of the viscous dissipation in turbulent flow simulation is examined. To this end, we will calculate here the two dimensional homogeneous isotropic turbulence, where the stretching term of the vorticity equation vanishes, and the direct assessment of the diffusion/dissipation process is possible. The results are compared with DNS, and the representation of spectra as well as the energy decay rates is examined.

${ }^{*}$ Received 27 Dec., 2006 (No. T-04-0256) Japanese Original: Trans. Jpn. Soc. Mech. Eng., Vol.71, No.701, B (2005), pp.23-29 (Received 17 Mar., 2004) [DOI: 10.1299/jfst.2.248] 


\section{Vortex Methods}

\subsection{Vortex Element Model}

The vorticity at an arbitrary point in the flow field is expressed by the total effect of all vortex elements in the flow as

$$
\omega(\mathbf{x})=\sum_{j} \omega_{j}^{s} f_{j}^{\omega}
$$

The $\omega_{j}^{s}$ and $f_{j}^{\omega}$ represent the vorticity strength and cutoff function of the $j$ th element respectively, and can be written in the following form,

$$
\begin{aligned}
& \omega_{j}^{s}=\omega\left(\mathbf{x}_{j}\right) \delta s_{j}, \\
& f_{j}^{\omega}=\frac{\exp \left(-\frac{r^{2}}{2 \epsilon_{j}^{2}}\right)}{2 \pi \epsilon_{j}^{2}},
\end{aligned}
$$

where $\delta s_{j}$ is the area of the vortex element, $r_{j}$ is the distance from $\mathbf{x}$ to the $j$ th particle, and $\epsilon_{j}$ is the core radius. The Gaussian function ${ }^{(4)}$ was used for the cutoff function.

The velocity at $\mathbf{x}$ is calculated by the Biot-Savart law

$$
\mathbf{u}(\mathbf{x})=-\sum_{j} \frac{\mathbf{r}_{j} \times \omega_{j}^{s} f_{j}^{u}}{r_{j}^{2}}
$$

where $\mathbf{r}_{j}$ is the relative position vector between $\mathbf{x}$ and the $j$ th element. $f_{j}^{u}$ is the cutoff function for the velocity calculation

$$
f_{j}^{u}=\frac{1-\exp \left(-\frac{r_{j}^{2}}{2 \epsilon_{j}^{2}}\right)}{2 \pi},
$$

which is similar to $f_{j}^{\omega}$.

The position and strength of the vortex elements are determined by integrating the following equations over time

$$
\begin{aligned}
\frac{d \mathbf{x}_{i}}{d t} & =\mathbf{u}_{i}, \\
\frac{d \omega_{i}^{s}}{d t} & =\left(\nu \nabla^{2} \omega^{s}\right)_{i} .
\end{aligned}
$$

The right hand side of Eq. (7) represents the diffusion of vorticity strength due to molecular viscosity, where $v$ is the kinematic viscosity. The question is whether this term will account for the energy dissipation in turbulent simulations or not. We will discuss below how this term is actually handled.

\subsection{Viscous Diffusion Model}

Numerous viscous diffusion models ${ }^{(5)}$ have been proposed for vortex methods. Here, we will focus on the core spreading method ${ }^{(6)}$, which is easy to handle and has been used recently in many engineering applications. Our particular interest is the problem it faces in turbulence simulations. On the other hand, the particle strength exchange (PSE) is more consistent with the continuum approach, but requires frequent re-meshing in order to maintain accuracy. This deprives the vortex method of its essential quality. In the present study we use the MPS Laplacian model, which has the qualities of the PSE, but is also grid-free. We will give a brief introduction to the two methods below.

2.2.1. Core Spreading Model The core spreading method ${ }^{(6)}$ is formulated by considering the diffusion of a single vortex element, which is expressed by the heat kernel. Its calculation cost is low and its algorithm is simple. By introducing this model, we calculate the expansion rate of the core radius used in Eqs. (3) and (5), to account for Eq. (7). The expansion rate is calculated by

$$
\frac{d \epsilon_{i}}{d t}=\frac{4 v}{2 \epsilon_{i}}
$$




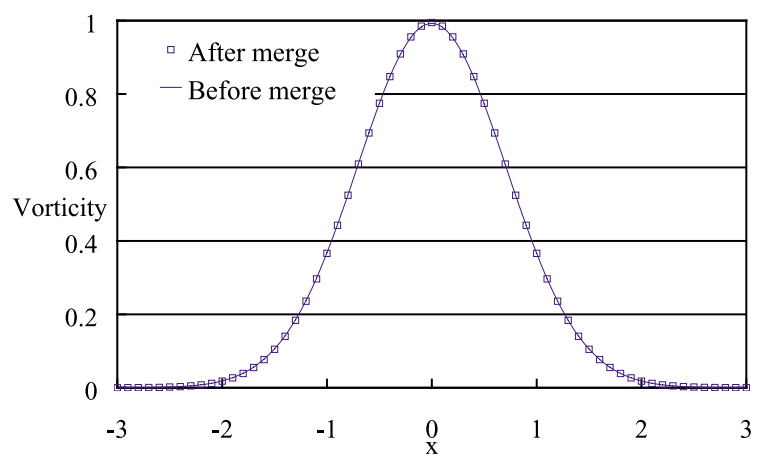

Fig. 1 Vorticity profile before and after the merge process.

The core spreading model calculates the diffusion without any interaction between the surrounding elements. As will be shown later, this causes the higher wave numbers of the energy spectrum to decay at a prominent rate compared to lower wave numbers.

2.2.2. MPS Laplacian Model In the Moving Particle Semi-implicit (MPS) by Koshizuka $\& \mathrm{Oka}^{(7)}$, the diffusion is expressed by a Laplacian model

$$
\left(v \nabla^{2} \omega^{s}\right)_{i}=\frac{4 v}{\lambda_{i} \bar{n}} \sum_{j \neq i}\left[\left(\omega_{j}^{s}-\omega_{i}^{s}\right) w\left(r_{i j}\right)\right] .
$$

$\lambda_{i}$ is a function which controls the particle density, and can be written as

$$
\lambda_{i}=\frac{1}{n_{i}} \sum_{j \neq i}\left[r_{i j}^{2} w\left(r_{i j}\right)\right]
$$

$\lambda_{i}$ is calculated for all vortex elements at every time step. $n_{i}$ in Eq. (10) represents the particle density and is given by

$$
n_{i}=\sum_{j \neq i} w\left(r_{i j}\right)
$$

The $\bar{n}$ in Eq. (9) is the average of $n_{i}$ for every time step.

For the weight function $w\left(r_{i j}\right)$, we use the original form proposed by Koshizuka \& Oka ${ }^{(7)}$

$$
w\left(r_{i j}\right)=\left\{\begin{aligned}
\frac{r_{e}}{r_{i}}-1 & \left(r_{i j} \leq r_{e}\right), \\
0 & \left(r_{i j}>r_{e}\right),
\end{aligned}\right.
$$

where $r_{i j}$ is the distance between elements and $r_{e}$ is the threshold of interaction. This threshold is a constant number which is affected by the particle density.

\subsection{Merging and Insertion Process}

Generally, the density of vortex elements in a vortex method calculation becomes uneven as the calculation proceeds. The MPS Laplacian model previously described is strongly affected by the particle density. In order to calculate the diffusion term accurately, the particles must be evenly distributed to some extent. In the present calculation, the particles were merged in the dense regions, and were inserted in the depleted regions.

Upon merging the vortex elements, the change in the distribution of vorticity before and after the merging process must be minimized in order to conserve the energy throughout the domain. We first search for a pair of vortex elements whose distance and vortex strength difference are below a certain threshold, and then merge them. This is done for all elements at every time step. The merged vortex element has vortex strength equal to the sum of the pair before merging, and the position is at the midpoint of the pair. Furthermore, the core radius $\epsilon$ is constant for all particles and is not changed by the merging process. The vorticity distribution before and after this merging process are shown in Fig. 1. When enstrophy was integrated over a radius of $3 \epsilon$ from the center of the merged element, the normalized difference before and after the merging was approximately $3 \times 10^{-3}$. 


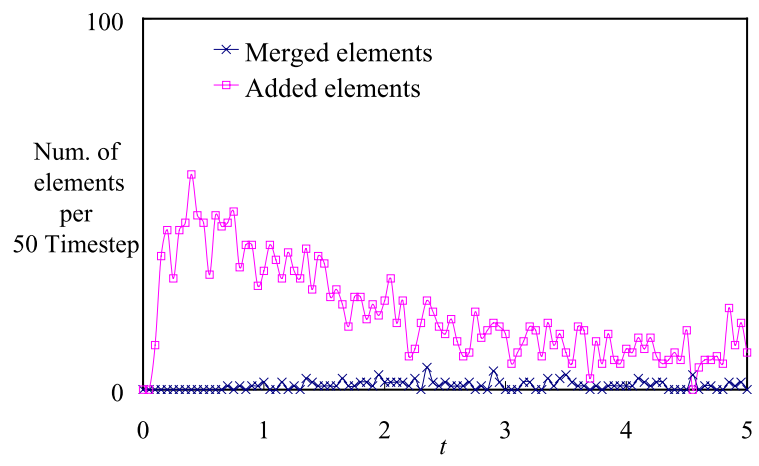

Fig. 2 Variations in number of merged and added elements.

Meanwhile, for the insertion of particles we must first find the depleted regions. This is done by dividing the calculation region into square boxes equal to the number of particles. We monitor the number of particles in each box, and when it becomes zero we insert a particle with zero vorticity at the center of the box. This insertion process does not change the vorticity distribution; hence, the energy is conserved.

We sum the number of elements which were merged or added within a 50 step time frame, and plot the time variation for 100 of these time frames in Fig. 2. The number of merged and inserted particles shows an asymptotic behavior to some constant value. It is worth noting that particles were merged only once every 50 time steps, and this implies that the total energy is conserved quite well. In the following calculation, the number of particles increased 1.5 times for a calculation of 5000 time steps.

\section{Two-Dimensional Homogeneous Isotropic Turbulence}

\subsection{Initial Condition}

We initialize our calculation using a prescribed energy spectrum given by Tatsumi \& Yanase $^{(8)}$ :

$$
E(k)=\frac{\overline{u_{0}^{2}} k^{3}}{k_{0}^{4}} \exp \left(-\frac{k^{2}}{k_{0}^{2}}\right) .
$$

$u_{0}$ is the velocity fluctuation and $k_{0}$ is the peak frequency of the spectrum, which is $k_{0}=4$ for the present calculation.

For the initialization of the vortex method calculation, we first calculate the vorticity field on a uniform grid in the $x-y$ plane from the prescribed energy spectrum in Eq. (13). Then, we place the discrete vortex elements on the grid and use the vorticity at the corresponding grid points as the initial vortex strength of the elements. We then multiply the initial vortex strength of all particles with a constant to match the total kinetic energy with the prescribed spectrum.

The calculation domain is a square box with side length $2 \pi$, and is periodic in all three directions. The Reynolds number based on the RMS value of the initial velocity and the side length of the domain is 1300 and 260 for the two cases we investigated.

\subsection{Boundary Condition}

The far field has a considerable effect on vortex method calculations, and the periodic boundary condition requires special attention. In a 3-D vortex method calculation of homogeneous isotropic turbulence ${ }^{(9)}$, an extraordinary oscillation was observed in the higher wave numbers, which was found to be a consequence of inappropriate boundary conditions. In the present investigation, we use a finite number of periodic images to approximate the effect of the far field. The accuracy of this procedure is strongly affected by the number of periodic images so we will first check this. 


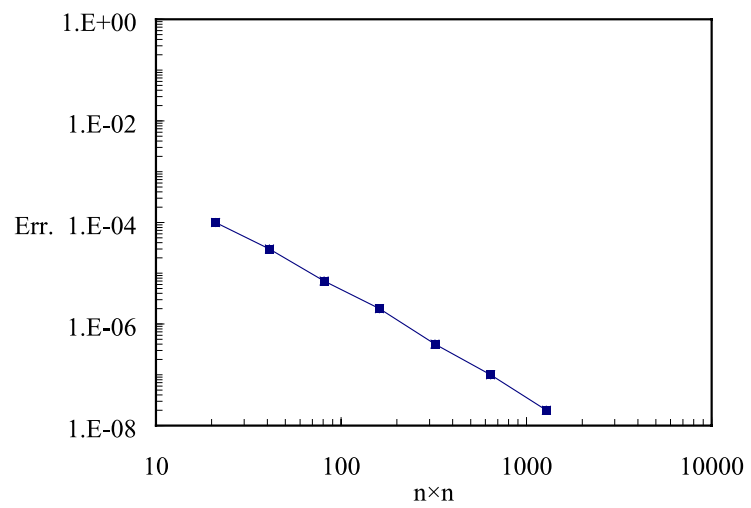

Fig. 3 Influence of the number of computation region on approximation of the periodic boundary condition.

Fig. 3 shows the relative error of induced velocity on a $128^{2}$ grid for a different number of periodic images $n$. We use the case where $n=2561$ as the reference velocity, and the error is given by the square norm of the difference of the two velocity fields. The results show an asymptotic behavior, and the relative error is $O\left(10^{-6}\right)$ for $81 \times 81$. For this reason, we will use $81 \times 81$ regions, including the original one, to approximate the effect of the periodic boundary conditions.

\subsection{Fast Algorithms}

We use the fast multipole method (FMM) ${ }^{(10)}$ to reduce the calculation cost of the vortex method from $N^{2}$ to $N$, where $N$ is the number of vortex elements. The test runs for the FMM were performed using $21 \times 21$ regions rather than $81 \times 81$. Our test calculations show that when the order of the approximation function is 15 , the square norm error is $O\left(10^{-7}\right)$, and thus we set the order to this value. Under these conditions, a run for $64 \times 64$ particles per region showed a 1020 fold acceleration compared to a direct calculation. The vortex method calculation takes twice as much calculation time as compared to the spectral DNS described in the following section.

\subsection{DNS for Reference}

We have conducted a Fourier-Galerkin spectral method calculation as a reference. By applying the Fourier expansion to the wave number $\mathbf{k}$ of the velocity and vorticity field, the vorticity transport equation becomes

$$
\frac{\partial \tilde{\omega}(\mathbf{k}, t)}{\partial t}=i \mathbf{k} \times(\mathbf{u} \widetilde{\times} \omega)(\mathbf{k}, t)-\nu \mathbf{k}^{2} \tilde{\omega}(\mathbf{k}, t) .
$$

This equation is solved for the same domain as the vortex method with periodic boundary conditions. The forth order Runge-Kutta was used for time stepping, and the calculation was performed on a $256^{2}$ grid. The aliasing error was removed by the 3/2-rule.

\section{Results and Discussion}

\subsection{Calculation Condition}

The following results were calculated by a second-order Adams-Bashforth method for Eqs. (6) and (7), and the viscous diffusion term was calculated explicitly. However, the core spreading calculation Eq. (8) was performed by using an explicit Euler method. The time step size is $\Delta t=10^{-3}$, and the number of time steps reached 5000 . The total calculation time spans 10 eddy turnover time units.

The particles were initially positioned on a $64 \times 64$ grid, and the initial core radius $\epsilon_{0}$ was varied against the element distance $r_{0}$ to check the effect on the energy decay. The threshold for the MPS Laplacian interaction $r_{e}$ was set to 3 times the initial particle distance. The thresh- 


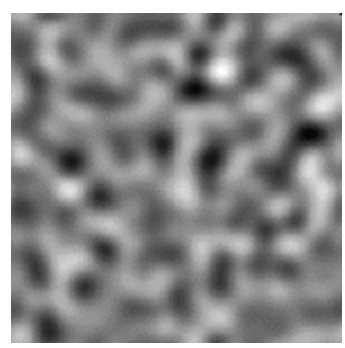

(a) Initial condition

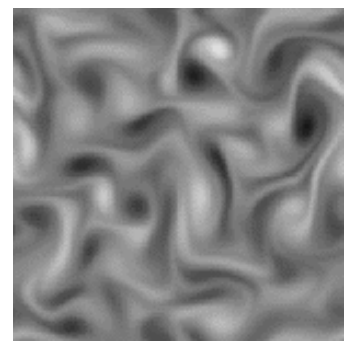

(b) DNS t $=0.5$

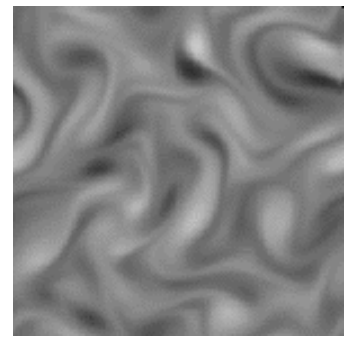

(c) DNS t $=1.0$

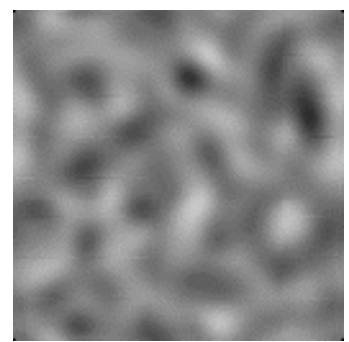

(d) $\mathrm{CS} t=0.5$

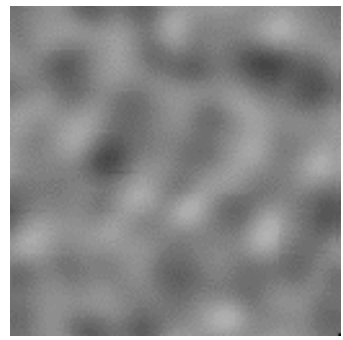

(e) CS $\mathrm{t}=1.0$

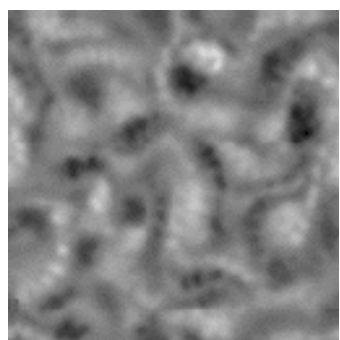

(f) MPS t $=0.5$

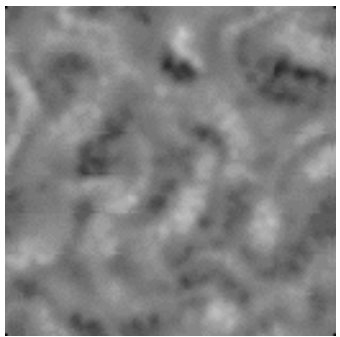

(g) MPS $\mathrm{t}=1.0$

Fig. 4 Distribution of vorticity. Black and white colors correspond to positive and negative vorticity, respectively. Scale is arbitrary but same for all figures.

old for the merging process are $0.1 \epsilon$ and $5 \%$ for the particle distance and relative difference of vortex strength, respectively.

\subsection{Vorticity Distribution}

The vorticity fields for the calculation with an initial $R e=1300$ are shown in Fig. 4 to compare the qualitative behavior. (a) is the initial condition, (b) and (c) are DNS results, (d) and (e) are for core spreading, and (f) and (g) are for the MPS Laplacian model at $t=0.5$ and $t=1.0$, respectively. The plots are for the entire calculation domain, where the DNS employs $256^{2}$ grid points while the vortex methods $128^{2}$. For the vortex methods, the values on the $128^{2}$ grid were calculated from the vorticity on discrete points by making use of Eq. (1). The grey regions indicate $\omega=0$, and the black and white represent regions of positive and negative vorticity, respectively.

If we look at the DNS results, the initially random vorticity starts to form structures at $t=0.5$ as shown in (b). At $t=1.0$, the contrast of color contour becomes less distinct, which indicates that the movement decays throughout the flow field.

The results of the core spreading method shown in (d) and (e) are blurry compared to the initial condition, but show only weak structural behavior. The pattern of the vorticity field only blurs in time, and the shape of the structures seems to remain constant. On the other 


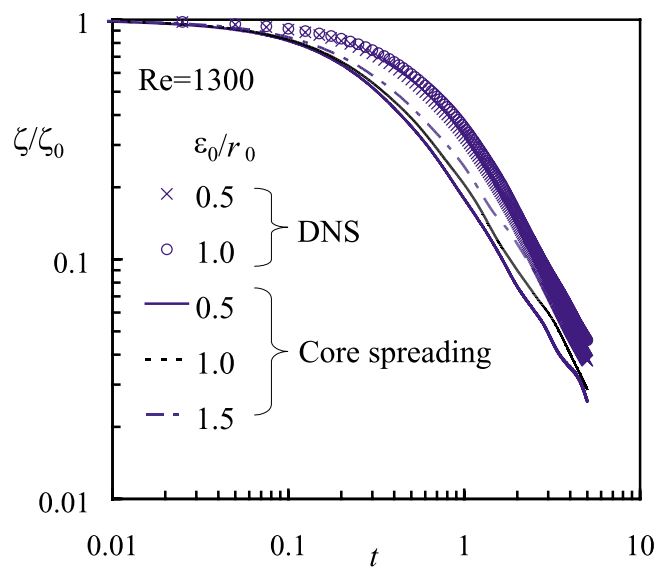

Fig. 5 Decay of enstrophy for CS and DNS at $R e=1300$.

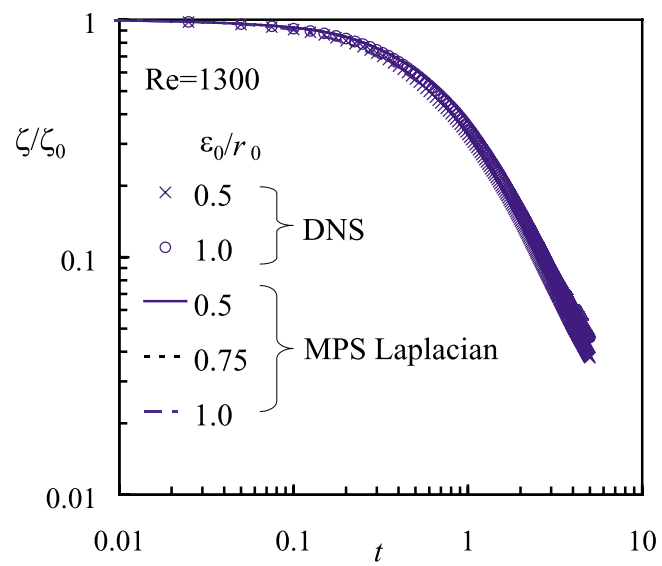

Fig. 6 Decay of enstrophy for MPS and DNS at $R e=1300$.

hand, the MPS Laplacian results shown in (f) and (g) are similar to the DNS in the sense that they show stretching of the large scale structures for $t=0.5$. Also, the contrast is sharper compared to the core spreading method. The fact that the large structures retain their form while the contrast fades is also quite similar to the DNS results.

The qualitative characteristics mentioned above appear as a notable difference in the energy spectrum, as we will show below.

\subsection{Decay of Enstrophy}

The time evolution of the spatially integrated enstrophy is examined in order to evaluate the dissipation of energy. The enstrophy is calculated from the vorticity by

$$
\zeta=\frac{1}{2} \int \omega^{2} d s .
$$

The vortex method results are evaluated on a $128 \times 128$ grid by using Eq. (1), as mentioned earlier.

The decay of enstrophy for the DNS and core spreading method are shown in Fig. 5. The vertical axis is normalized by the enstrophy $\zeta_{0}$ at $t=0$. Our DNS results agree qualitatively with that of Tatsumi \& Yanase ${ }^{(8)}$. The core spreading method decays much faster than the DNS. It is quite possible that the initial core radius $\epsilon_{0}$ has some kind of effect, although changing this value from $0.5 r_{0}$ to $1.5 r_{0}$ did not seem to improve the initial discordance. The initial conditions of the DNS were also reconfigured to reflect the change of $\epsilon_{0}$, but the results were not affected by this. On the other hand, Fig. 6 shows good agreement between the MPS Laplacian model and the DNS. The results are not affected by the change in $\epsilon_{0}$. 


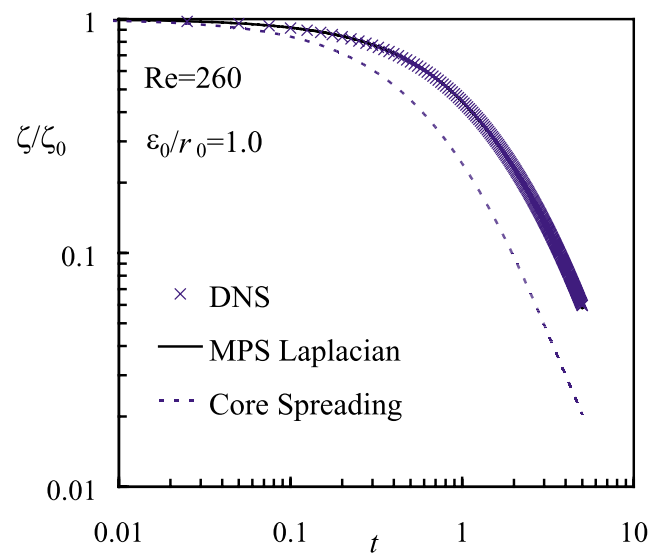

Fig. 7 Decay of enstrophy at $R e=260$.

The tendency of the above results are the same for the initial Reynolds number $R e=260$ as shown in Fig. 7. The MPS Laplacian model is in good accordance with the DNS as to where the core spreading model shows a large difference. From these observations, it is fair to say that the viscous diffusion model has a strong effect on the determination of the energy dissipation.

\subsection{Energy Spectrum}

Now we will compare the energy spectrum of the two models with that of the DNS. Fig. 8 shows the results of the core spreading method, and Fig. 9 is for the MPS Laplacian. The initial Reynolds number is $R e=1300$ and the number of vortex elements is $64 \times 64$ for both cases. The core radius was chosen to yield the closest results to the DNS, and were $\epsilon_{0} / r_{0}=1.5$ for the core spreading method and 0.75 for the MPS Laplacian model.

The DNS results first show an energy transfer from the lower to higher wave numbers, then the energy decays gradually throughout the entire wave band. The core spreading method retains its initial spectral form, and decays without showing any sign of an energy cascade. In contrast, the MPS Laplacian model collapses the DNS results well for both the cascade and decay process.

The above observations match that of the vorticity distribution shown in Fig. 4, i.e. the core spreading method directly decays from the initial distribution, while the MPS Laplacian forms large structures and then decays, as does the DNS. The energy transfer from low to high wave numbers corresponds to the thin filaments in between the large structures in the vorticity distribution.

The core spreading method calculates the diffusion as an isolated process for individual vortex elements, whereas the MPS Laplacian model calculates the interaction of elements and also merges and inserts elements to maintain an even distribution. By summarizing the above results, we can see that the latter features lead to results closer to the DNS by generating thin structures between large ones so that high wave number components are produced. From these results, it is quite obvious that the treatment of viscous diffusion models plays an important role in the dissipation process of vortex methods.

For this particular case of a two-dimensional homogeneous isotropic turbulence calculation, the Reynolds number does not influence the necessary number of vortex elements as much as the grid points for the continuum approach. In general, the smallest scale in a turbulent flow is a function of the Reynolds number, so the spatial resolution required will increase with the Reynolds number. Consequently, the grid size reflects the smallest scale resolvable in Navier-Stokes based continuum approaches. However, for the vortex methods the elements size and the element number do not have such a direct relation. For example in Fig. 9, the MPS Laplacian model omits the higher wave numbers, but the decay of enstrophy are still in 


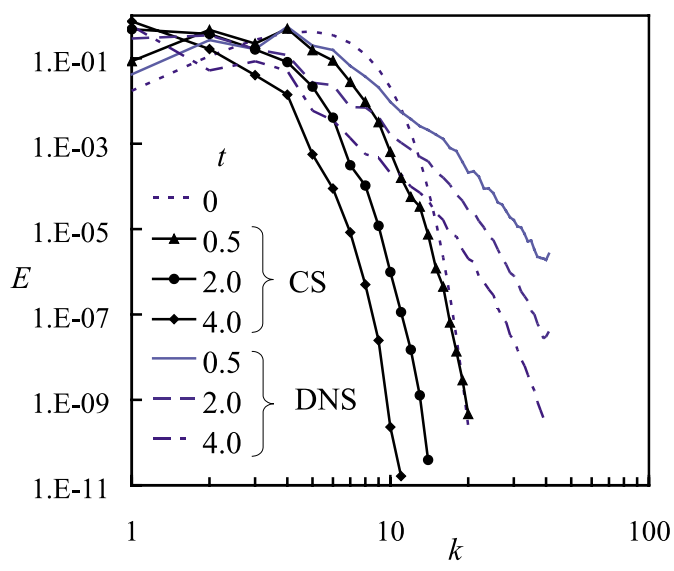

Fig. 8 Energy spectra of CS and DNS at $R e=1300$.

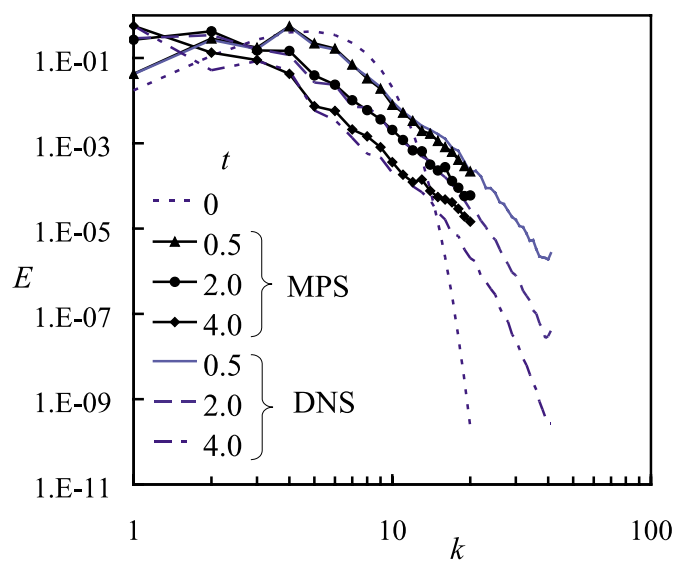

Fig. 9 Energy spectra of MPS and DNS at $R e=1300$.

good accordance. This implies that the accordance of vortex methods and Navier-Stokes simulations depends more on the viscous diffusion models than the number of vortex elements.

\section{Conclusions}

The viscous diffusion models in the vortex method are examined by calculating twodimensional homogeneous isotropic turbulence where stretching term is absent. The results were investigated both qualitatively and quantitatively, by comparing with a DNS performed under the equivalent condition.

The core spreading method, which calculates the diffusion of individual vortex elements, does not have the ability to predict the time evolution of the enstrophy and energy spectrum. Meanwhile, the MPS Laplacian model, which calculates the interaction of vortex elements along with merging and insertion to achieve uniform particle density, agrees well with DNS results.

The application of vortex methods to calculations of three-dimensional flows should deserve more attentions because the approximation of stretching term may mask the deficit of diffusion models. The systematic assessment of vortex methods in three-dimensional turbulent flows is desired.

\section{Acknowledgments}

This study was supported by a Grant-in-Aid for the 21st century COE Program "System Design: Paradigm Shift from Intelligence to Life" from the Ministry of Education, Culture, Sports, Science and Technology of Japan. 


\section{References}

( 1 ) Ojima, A. and Kamemoto, K., Numerical simulation of unsteady flow through a horizontal axis wind turbine by a vortex method, The Second International Conference on Vortex Methods (2001), 173-180.

( 2 ) Kiya, M. and Ishii, H., Vortex interacton and Kolmogorov spectrum, FDR 8 (1991), 73-83.

( 3 ) Cottet, G.H., Michaux, B., Ossia, S., and VanderLinden, G., A comparison of spectral and vortex methods in three-dimensional incompressible flows, J. Comp. Phys. 175 (2002), 702-712.

( 4 ) Winkelmans, G.S., and Leonard, A., Contributions to vortex particle methods for the computation of three-dimensional incompressible unsteady flows, J. Comp. Phys. 109 (1993), 247-273.

( 5 ) Cottet, G.H. and Koumoutsakos, P.D., Vortex Methods, Cambridge University Press (2000).

( 6 ) Leonard, A., Vortex methods for flow simulation, J. Comp. Phys. 37 (1980), 289-335.

( 7 ) Koshizuka, S. and Oka, Y., Moving-particle semi-implicit method for fragmentation of incompressible fluid, Nucl. Sci. Eng. 123 (1996), 421-434.

( 8 ) Tatsumi, T. and Yanase, S., The modified cumulant expansion for two-dimensional isotropic turbulence, J. Fluid Mech. 110 (1981), 475-496.

( 9 ) Totsuka, Y. and Obi, S., Turbulent flow analysis by a three dimensional vortex method, The 14th CFD symposium (2000), E09-4. (in Japanese)

(10) Greengard, L. and Rokhlin, V., A fast algorithm for particle simulations, J. Comp. Phys. 73 (1987), 325-348. 